\title{
DEGENERATE DWARFS WITH LIQUEFYING CORES
}

(Abstract)

\author{
UMBERTO DE ANGELIS \\ Astronomical Observatory, Naples, Italy
}

The problem of phase transitions in hot white dwarf matter has been recently investigated and the results applied to follow the cooling of degenerate dwarfs whose core undergoes liquefaction and to build liquefying sequences on the HR diagram.

Gas-liquid phase transitions are found to occur at $\Gamma \simeq 1$ and the transition points are found to be well fitted by the rule

$$
T_{L}=1.4 \times 10^{5} Z^{5 / 3} \varrho^{1 / 3},
$$

where $T_{L}$ is the liquefaction temperature, $\varrho$ the corresponding density and $Z$ the chemical composition.

Equation (1) gives a liquefaction temperature about $100 \mathrm{~K}$ higher than the melting temperature (for the same $\varrho, Z$ ) as given by Lindemann's melting rule

$$
T_{m}=3.7 \times 10^{3} Z^{5 / 3} \varrho^{1 / 3}
$$

so that crystallization will set in at $\Gamma \simeq 100$.

Assuming a star model with a degenerate, hot, isothermal core $(\varrho, T, Z$ in the liquefying range) and an envelope acting as a thermal blanket, an opacity was derived in the form:

$$
k=k_{0} \varrho^{n} T^{-S}
$$

and then the luminosity law for such a model could be written in the form

$$
L=L_{0} M \varrho_{C}^{-0.4} T_{C}^{3.4}
$$

where the subscript $C$ indicates central values and $M$ is the star's mass.

By combining Equations (1) and (2) a luminosity law for stars with liquefying cores results

$$
L=L_{0}^{\prime} M Z^{1.9} T_{C}^{1.45}
$$

For such models a central temperature-effective temperature relation was also obtained

$$
T_{e}=\text { constant } \times M^{5 / 12}\left(Z T_{c}\right)^{1 / 2}
$$

Equations (3) and (4) were then used to build liquefying sequences on the HR diagram and these came out to be in the region of the diagram dense with observed nuclei of planetary nebulae. 
Further evidence suggesting a possible identification of such models as central stars of planetary nebulae and an overall evolutionary picture from the nebula ejection to a crystalline white dwarf has been considered in (3) and (4).

\section{References}

De Cesare, L., Forlani, A., and Platania, G.: 1973, Astrophys. Space Sci., 21, 461.

De Angelis, U., De Cesare, L., Forlani, A., and Platania, G.: 1973, Astrophys. Space Sci. $20,875$.

De Angelis, U. De Cesare, L., Forlani, A., and Platania, G.: 1973, Nature Phys. Sci. 244, 133.

De Angelis, U., De Cesare, L., Forlani, A., and Platania, G.: 1973, Astrophys. Space Sci. 20, 886. 